\title{
The effect of inspiratory and expiratory loads on abdominal muscle activity during breathing in subjects "at risk" for the development of chronic obstructive pulmonary disease and healthy
}

\author{
António Mesquita Montes ${ }^{\mathrm{a}, \mathrm{b}}$, \\ Carlos Crasto ${ }^{\mathrm{a}}$, \\ Cristina Argel de Melo ${ }^{a}$, \\ Rita Santos ${ }^{a}$, \\ Susana Pereira ${ }^{\mathrm{c}}$, \\ João Paulo Vilas-Boas ${ }^{\text {b }}$
}

\author{
a Department of Physiotherapy, and Activity and Human Movement Study Center (CEMAH), School of Allied Health Technologies, Polytechnic Institute of Porto, Rua Dr. \\ António Bernardino de Almeida 400, 4200-072 Porto, Portugal \\ ${ }^{\mathrm{b}}$ Faculty of Sport, CIFI2D, and Porto Biomechanics Laboratory (LABIOMEP), University of Porto, Rua Dr. Plácido Costa 91, 4200-450 Porto, Portugal \\ ${ }^{\mathrm{c}}$ Cardiovascular, Respiratory and Sleep Technician, Private Practice, Portugal
}

A B S T R A C T

\begin{abstract}
The abdominal muscle activity has been shown to be variable in subjects with chronic obstructive pulmonary disease (COPD) when respiratory demand increases and their recruitment pattern may change the mechanics, as well as the work and cost of breathing. The scientific evidence in subjects "at risk" for the development of COPD may be important to understand the natural history of this disease. This study aims to evaluate the effect of inspiratory and expiratory loads on the abdominal muscle activity during breathing in subjects "at risk" for the development of COPD and healthy. Thirty-one volunteers, divided in "At Risk" for COPD ( $\mathrm{n}=17 ; 47.71 \pm 5.11$ years) and Healthy ( $\mathrm{n}=14 ; 48.21 \pm 6.87$ years) groups, breathed at the same rhythm without load and with $10 \%$ of the maximal inspiratory or expiratory pres-sures, in standing. Surface electromyography was performed to assess the activation intensity of rectus abdominis (RA), external oblique and transversus abdominis/internal oblique ( $\mathrm{Tr} A / \mathrm{IO})$ muscles, during inspiration and expiration. During inspiration, in "At Risk" for COPD group, RA muscle activation was higher with loaded expiration $(p=0.016)$; however, in Healthy group it was observed a higher activation of external oblique and TrA/IO muscles $(p<0.050)$. During expiration, while in "At Risk" for COPD group, RA muscle activation was higher with loaded inspiration $(p=0.009)$, in Healthy group TrA/IO muscle showed a higher activation $(p=0.025)$. Subjects "at risk" for the development of COPD seemed to have a specific recruitment of the superficial layer of ventrolateral abdominal wall for the mechanics of breathing.
\end{abstract}

Keywords: GOLD “Stage 0" Respiration Respiratory loads Core abdominal

\section{Introduction}

Chronic obstructive pulmonary disease (COPD) is described as the presence of persistent airflow limitation that is usually progressive and associated with an enhanced chronic inflammatory response in the airways (Global Initiative for Chronic Obstructive Lung Disease, 2016; Vestbo et al., 2013). The chronic inflammation causes structural changes and narrowing of the small airways, as well as the loss of alveolar attachments to the small airways and decreases lung elastic recoil. These changes diminish the ability of airways to remain open during expiration (Baraldo et al., 2012). Although the primary physiological defect, inherent to the subjects with COPD, is the expiratory flow limitation, an important mechanical consequence of this phenomenon is the incomplete lung emptying during resting tidal breathing - lung hyperinflation (O'Donnell, 2001). From a mechanical point of view, the lung hyperinflation shortens and flattens the diaphragm muscle and negatively modifies its length-tension relationship. As a result, the diaphragm muscle reduces the flow and pressure-generating capacity (Gea et al., 2015). Therefore, neural drive (increased firing rate and recruitment) to the diaphragm muscle (De Troyer et al., 1997; Gorini et al., 1990) and activity of parasternal intercostal and scalene muscles (Gandevia et al., 1996) are increased in COPD, during breathing at rest or when ventilation increases. Furthermore, the expiratory contraction of abdominal muscles in subjects with COPD may be an "automatic" response to the increased work 
of breathing and ventilatory stimulation, even during resting breathing (Martinez et al., 1990; Ninane et al., 1992). It has been proposed that an increased abdominal muscle activity is an appropriated response to assist inspiratory muscles, reducing the endexpiratory lung volume (Aliverti et al., 1997). This action of abdominal muscles changes diaphragmatic configuration, optimizing its length-tension characteristics (De Troyer and Estenne, 1988), or it allows the release of stored elastic energy at the onset of inspiration (Aliverti et al., 1997). Nevertheless, the abdominal muscle activity has been shown to be variable in subjects with COPD when respiratory demand increases (Laveneziana et al., 2014), and their recruitment pattern may change the mechanics, as well as the work and cost of breathing (Aliverti and Macklem, 2008).

The impact of different respiratory loads on abdominal muscle activity, during both breathing phases, for mechanics of breathing is not yet clear in subjects "at risk" for the development of COPD (presence of chronic respiratory symptoms, in addiction to some evidence of impaired lung function) (Rodriguez-Roisin et al., 2016). Although many subjects in COPD "Stage 0" do not necessarily progress to chronic airflow limitation, unobstructed smokers with other chronic respiratory symptoms (such as dyspnea, wheeze and limited physical activity) experience significant morbidity and need health care resources, which represents a potential clinical entity (de Marco et al., 2007; de Oca et al., 2012; Mannino et al., 2006; Stavem et al., 2006). The scientific evidence in these subjects may be important to understand the natural history of COPD. The aim of the present study was to evaluate the effect of inspiratory and expiratory loads on the abdominal muscle activity during breathing in subjects "at risk" for the development of COPD and healthy. Specifically, it was analysed the activation intensity of rectus abdominis (RA), external oblique (EO) and transversus abdominis/internal oblique ( $\mathrm{Tr} A / \mathrm{IO})$ muscles, during inspiration and expiration, without respiratory load and with inspiratory or expiratory loads. To our knowledge, the possible changes on the mechanics of breathing associated with early lung disease in smokers have not been investigated. Therefore, we hypothesized that the recruitment pattern of abdominal muscles would be different in subjects "at risk" for the development of COPD, when respiratory demand increases.

\section{Methods}

\subsection{Sample}

The study followed a cross-sectional design with a sample composed by thirty-one volunteers of an higher education institution: seventeen subjects "at risk" for the development of COPD - "At Risk" for COPD group; and fourteen healthy subjects - Healthy group. Sociodemographic, anthropometric and body composition data were similar between groups (Table 1). Participants had not participated in aerobic physical activities with a moderate intensity (a minimum of 30 min on five days a week) and/or aerobic physical activities with a vigorous intensity (a minimum of 20 min on 3 days a week), for a period exceeding one year (Thompson, 2014). As inclusion criteria for the "At Risk" for COPD group, participants had dyspnea, chronic cough and sputum production at least for three months in two consecutive years, as well as history of exposure to risk factors (namely smoking habits at least for fifteen years) (Rodriguez-Roisin et al., 2016). Moreover, these participants had to have Grade 1 or more in the Modified British Medical Research Council (mMRC) questionnaire and one point or more, out of five points, in the first four items of the COPD Assessment Test (CAT) (presence of cough, mucus, chest tightness and breathlessness) (Global Initiative for Chronic Obstructive Lung Disease, 2016). Exclusion criteria for both groups included chronic
Table 1

“At Risk" for COPD and Healthy groups' characterization: sociodemographic, anthropometric and body composition data, with mean and standard deviation. $p$ values for significant differences between groups are also presented.

\begin{tabular}{llll}
\hline & $\begin{array}{l}\text { "At Risk" for } \\
\text { COPD group } \\
(\mathrm{n}=17)\end{array}$ & $\begin{array}{l}\text { Healthy } \\
\text { group } \\
(\mathrm{n}=14)\end{array}$ & $\begin{array}{l}\text { Between groups } \\
\text { comparison } \\
(p \text { value })\end{array}$ \\
\hline $\begin{array}{l}\text { Sociodemographic and anthropometric data } \\
\text { Gender (n male) }\end{array}$ & 5 & 6 & \\
Age (years) & $47.71 \pm 5.11$ & $48.21 \pm 6.87$ & 0.477 \\
Body mass (kg) & $70.85 \pm 14.37$ & $79.65 \pm 15.28$ & 0.110 \\
Height (m) & $1.67 \pm 0.11$ & $1.67 \pm 0.10$ & 0.917 \\
Body composition data & & & \\
Body fat (\%) & $28.85 \pm 9.29$ & $32.66 \pm 8.91$ & 0.256 \\
Total body water (\%) & $49.19 \pm 5.73$ & $48.13 \pm 4.93$ & 0.588 \\
Muscle mass (kg) & $48.51 \pm 11.21$ & $52.27 \pm 13.02$ & 0.395 \\
Bone mineral mass (kg) & $2.57 \pm 0.56$ & $2.76 \pm 0.63$ & 0.374 \\
Visceral fat & $7.12 \pm 3.14$ & $9.21 \pm 2.83$ & 0.063 \\
\hline
\end{tabular}

nonspecific lumbopelvic pain (recurrent episodes of lumbopelvic pain for a period longer than three months); scoliosis, length discrepancy of the lower limbs or other postural asymmetries; history of spinal, gynaecological or abdominal surgery in the previous year; neurological or inflammatory disorders; metabolic or chronic cardio-respiratory diseases; pregnancy or post-delivery in the previous six months; long-term corticosteroid therapy; and any conditions that may interfere with the data collection (American Thoracic Society/European Respiratory, 2002; Beith et al., 2001; Chanthapetch et al., 2009; Hermens et al., 2000; Mew, 2009; Miller et al., 2005; Reeve and Dilley, 2009). Each participant provided written informed consent, according to the Declaration of Helsinki. The anonymity of participants and the confidentiality of data were guaranteed. The Institutional Research Ethics Committee approved this study.

\subsection{Instruments}

\subsubsection{Surface electromyography (sEMG)}

sEMG was performed to assess the muscle activity of RA, EO and TrA/IO of the dominant hand side. The muscle activity was collected using the BioPlux research device (Plux wireless biosignals S.A., Arruda dos Vinhos, Portugal) with analogue channels of 12 bits and a sampling frequency of $1000 \mathrm{~Hz}$, using double differential electrode leads. Disposable, self-adhesive $\mathrm{Ag} / \mathrm{AgCl}$ dual snap electrodes (Noraxon Corporate, Scottsdale AZ, United States of America) were used for the sEMG. The electrode characteristics were $4 \times 2.2 \mathrm{~cm}$ of adhesive area, $1 \mathrm{~cm}$ diameter of each circular conductive area and $2 \mathrm{~cm}$ of inter-electrode distance. These electrodes were connected to bipolar active sensors emgPLUX (Plux wireless biosignals S.A., Arruda dos Vinhos, Portugal) with a gain of 1000 , an analogue filter at $25-500 \mathrm{~Hz}$ and a common-mode rejection ratio of $110 \mathrm{~dB}$. The reference electrode used was a disposable self-adhesive $\mathrm{Ag} / \mathrm{AgCl}$ snap electrode (Noraxon Corporate, Scottsdale AZ, United States of America) for the $\mathrm{SEMG}$, with $3.8 \mathrm{~cm}$ diameter of circular adhesive area and $1 \mathrm{~cm}$ diameter of circular conductive area. The sensors were Bluetooth connected through the sEMG device to a laptop. MonitorPlux software, version 2.0 (Plux wireless biosignals S.A., Arruda dos Vinhos, Portugal), was used to display and acquire the sEMG signal. An electrode impedance checker was used to assess the impedance level of skin (Noraxon Corporate, Scottsdale AZ, United States of America).

\subsubsection{Respiratory flow}

A respiratory flow transducer TSD117 - Medium Flow Trans 300 L.min-1 (Biopac Systems Inc., Goleta CA, United States of America) connected to an amplifier DA100C - General Purpose Transducer Amplifier Module (Biopac Systems Inc., Goleta CA, Uni- 
ted States of America), was used to detect both breathing phases. The respiratory flow was collected using the Biopac MP100WSW Data Acquisition System device (Biopac Systems Inc., Goleta CA, United States of America) with a sampling frequency of $100 \mathrm{~Hz}$. AcqKnowledge software, version 4.1 (Biopac Systems Inc., Goleta CA, United States of America), was used to display and acquire the respiratory flow signal.

\subsubsection{Maximal respiratory pressures and respiratory loads}

A respiratory pressure meter MicroRPM (CareFusion Corporation, San Diego CA, United States of America) was used to assess the maximal inspiratory (MIP) and expiratory (MEP) pressures. The values obtained were used to determine the inspiratory and expiratory loads, respectively. MEP quasi-static maximal manoeuvre was used to normalize the sEMG signal of abdominal muscles (maximal muscle activity of each muscle during breathing).

Flow independent one-way valve devices, Thresholds IMT breathing trainer - and PEP - positive expiratory pressure device (Respironics Inc., Murrysville PA, United State of America) were used to apply the inspiratory and expiratory loads, respectively. These devices were adapted to the respiratory flow transducer.

\subsection{Procedures}

\subsubsection{Sample selection and characterization}

An electronic questionnaire was delivered to all participants to verify the selection criteria and to collect sociodemographic information. Also, the mMRC and CAT were included in this questionnaire. Anthropometric and body mass composition measures were assessed in participants who met the participation criteria. Height ( $\mathrm{m}$ ) was measured using a seca 222 stadiometer with a precision of $1 \mathrm{~mm}$. Body mass ( $\mathrm{kg}$ ) and body mass composition - body fat (\%), total body water (\%), muscle mass ( $\mathrm{kg}$ ), bone mineral mass $(\mathrm{kg})$ and visceral fat - were assessed using a Tanita Ironman Inner Scan BC-549 body composition monitor with a precision of $1 \mathrm{~kg}$ and 1\% (Tanita - Monitoring Your Health, Amsterdam, Netherlands). To assess postural asymmetries, the lower limb length $(\mathrm{cm})$ was measured using a seca 201 tape with a precision of $1 \mathrm{~mm}$ (seca - Medical Scales and Measuring Systems, Hamburg, Germany) and the postural assessment was performed. These evaluations were performed to select the final sample. Women who were in luteal phase were contacted later for data collection.

A MasterScreen Body plethysmograph of volume-constant type (Jaeger - CareFusion Corporation, San Diego CA, United States of America) was used to record forced vital capacity and then to assess pulmonary function: forced expiratory volume in one second $\left(\mathrm{FEV}_{1}\right)$ /forced vital capacity (FVC); \% predicted of $\mathrm{FEV}_{1}, \mathrm{FVC}$, peak expiratory flow (PEF), forced expiratory flow at $75 \%$ $\left(\mathrm{FEF}_{75 \%}\right) / 50 \%\left(\mathrm{FEF}_{50 \%}\right) / 25 \%\left(\mathrm{FEF}_{25 \%}\right)$ of $\mathrm{FVC}$ and $\mathrm{FEF}_{25-75 \%}$. FVC manoeuvre (closed circuit method) was recorded with participants in sitting position, using a mouthpiece firmly held around the lips to prevent leakage and to support the cheeks, as well as a nasal clip to prevent nasal breathing. To assess this manoeuvre, a completely and rapidly inhalation was performed with a pause of one second at total lung capacity, followed by a maximally exhalation until no more air can be expelled while maintaining the upright posture. Each manoeuvre was encouraged verbally. A minimum of three manoeuvres was performed. To test result selection, three reproducible manoeuvres were recorded, according to Miller et al. (2005) standards. An expert cardiovascular, respiratory and sleep technician was responsible for this assessment.

\subsubsection{Data collection protocol}

The study procedures took place at a biomechanical laboratory and were performed in a controlled environment. To avoid interrater error, each researcher was responsible for an only one task.
To perform the sEMG, the hair was shaved and an abrasive cream was used to remove the dead cells from the skin's surface. Skin was then cleaned with isopropyl alcohol (70\%), removing its oiliness and holding the dead cells. An electrode impedance checker was used to make sure that the impedance levels were below $5 \mathrm{~K} \Omega$, thus ensuring a good acquisition of sEMG signal (Hermens et al., 2000). The self-adhesive electrodes were placed with participants in standing position, five minutes after the skin preparation. These electrodes were placed parallel to the muscle fibre orientation, according to the following references: RA, $2 \mathrm{~cm}$ lateral to the umbilicus, over the muscle mass; EO, Lateral to the RA and directly above the anterior superior iliac, halfway between the crest and ribs at a slightly oblique angle; $\operatorname{TrA} / \mathrm{IO}, 2 \mathrm{~cm}$ medially and below to the anterior superior iliac spine (in this local, $\operatorname{Tr} A$ and inferior internal oblique muscle fibres are mixed, so it is impossible to distinguish the surface electromyographic activity of both) (Criswell, 2011; Marshall and Murphy, 2003). The electrode placements were confirmed by palpation and muscle contraction. The reference electrode was placed in the anterior superior iliac spine of the contralateral hand dominant side. All electrodes were tested to control the cross-muscular signal (cross-talk), electrical noise and other interferences of sEMG signal (Hermens et al., 2000).

MIP and MEP were both performed with participants in standing position, using a disposable bacterial filter and mouthpiece firmly held around the lips to prevent leakage and to support the cheeks, as well as a disposable nasal clip to prevent nasal breathing (Biopac Systems Inc., Goleta CA, United States of America). To assess MIP, a forceful and maximal inspiration was performed the Muller manoeuvre - at residual volume; in turn, MEP was assessed through a forceful and maximal expiration - the Valsalva manoeuvre - at total lung capacity. Each manoeuvre was encouraged verbally. These manoeuvres were performed during a sixsecond period, with a resting time of three minutes. To calculate the respiratory load, three reproducible manoeuvres were recorded according to the American Thoracic Society/European Respiratory (2002) standards. It was used $10 \%$ of the best value of MIP and MEP for the inspiratory and expiratory loads, respectively.

All participants were subjected to three different tasks - breathing without respiratory load and with inspiratory and expiratory loads, in standing - in a single data collection moment. The data collection moment started with breathing without respiratory load. The order of inspiratory or expiratory loads was randomized. The participants were barefoot and had the upper limbs along the body, with feet shoulder-width apart and knees in loose pack position. An A3 paper sheet was used to outline the base of support, keeping it for all tasks. The participants kept their gaze in a horizontal direction and the respiratory flow transducer was kept perpendicular to the participant during all tasks. Disposable bacterial filter, mouthpiece and nose clip were also used. A single repetition of each task was performed for ten consecutive respiratory cycles, with a resting time of three minutes. The respiratory rhythm (inspiratory time: two seconds; expiratory time: four seconds) was marked through a recorded voice. This respiratory rhythm was previously defined in a pilot study. The participant experienced and get used to this externally paced respiratory rhythm prior to data collection.

After data collection, the electrodes were removed and a moisturizing cream was applied.

\subsubsection{Data processing}

A routine was developed in MatLab Student software (MathWorks, Pozuelo de Alarcon, Spain) to synchronize and process data. Firstly, the sEMG signal was converted into volts. It was applied to the sEMG signal a 2nd order digital filter Infinite Impulse Response - Butterworth, one of $30 \mathrm{~Hz}$ (high pass) and another of $500 \mathrm{~Hz}$ (low pass), to remove the electrical noise and/or cable movement and to 
remove the cardiac signal. Drake and Callaghan (2006) evaluated different techniques for removing electrocardiogram contamination from sEMG signal: high pass filter, amplitude and/or frequency subtraction methods. These authors reported that a butterworth high pass filter with $30 \mathrm{~Hz}$ cutoff provided the optimal balance between the ease of implementation, the time investment and the performance across different muscle contractions and heart rate levels. Finally, root mean square (RMS) to 10 samples was then calculated.

AcqKnowledge software, version 4.1, was used to analyse data. The abdominal muscle activity was analysed during inspiration and expiration, independently. These both breathing phases were identified through the respiratory flow transducer signal. For the ten respiratory cycles collected, the mean RMS of four central respiratory cycles of each muscle was analysed in each task, with a posterior analysis of its average .

The muscle activity collected during the MEP manoeuvre was used to normalize data of the abdominal muscles. The mean RMS of three central seconds of the expiratory phase of each muscle was analysed, and then the average of the mean RMS of three reproducible manoeuvres was calculated. The percentage of the activation intensity of each muscle was determined according to the following equation:

Muscle activation intensity $(\%)=\left(\frac{\text { mean RMS of each task }}{\text { RMS of the MEP }}\right) * 100$

\subsection{Statistical analysis}

IBM SPSS Statistics ${ }^{\circledR}$ software, version 20.0, (IBM Corporation, Armonk NY, United States of America) was used for the descriptive and inferential data analysis, with a significance level of 0.05 . Shapiro-Wilk test was used to test the normality of the data. Central tendency (mean) and dispersion (standard deviation) measures were used for the descriptive statistics. Chi-square was used to compare gender between groups ("At Risk" for COPD and Healthy). Student $t$-test was used to compare the age, anthropometric, body composition and pulmonary function data, as well as the percentage of muscle activation intensity, between groups. In each group, Repeated Measures Analysis of Variance was used to compare the percentage of muscle activation intensity between the different evaluation tasks (without respiratory load and with inspiratory or expiratory loads), during inspiration and expiration. Bonferroni correction was used for the post hoc analysis (Marôco, 2014). To quantify the effect size, the partial eta square $\left(\eta_{p}^{2}\right)$ values will be calculated using cut-off provided by Cohen: $\eta_{p}^{2}=0.01-$ small effect, $\eta_{p}^{2}=0.06$ - medium effect, and $\eta_{p}^{2}=0.14$ - large effect (Cohen, 1988).

\section{Results}

\subsection{Pulmonary function}

The forced expiratory volume in one second, peak expiratory flow and forced expiratory flow at $75 \% / 50 \% / 25 \% / 25-75 \%$ of FVC were significantly lower in "At Risk" for COPD group when compared to Healthy group $(p<0.050)$. No significant differences were found in the FVC between groups (Table 2 ).

\subsection{Muscle activity}

\subsubsection{Inspiration}

During inspiration, no significant differences were found in the activation intensity of all abdominal muscles between "At Risk" for COPD and Healthy groups during all tasks (Fig. 1).
Table 2

Pulmonary function data in "At Risk" for COPD and Healthy groups, with mean and standard deviation. $p$ values for significant differences between groups are also presented.

\begin{tabular}{llll}
\hline Pulmonary function & $\begin{array}{l}\text { “At Risk" for } \\
\text { COPD group } \\
(\mathrm{n}=17)\end{array}$ & $\begin{array}{l}\text { Healthy group } \\
(\mathrm{n}=14)\end{array}$ & $\begin{array}{l}\text { Between groups } \\
\text { comparison } \\
(p \text { value })\end{array}$ \\
\hline $\mathrm{FEV}_{1} / \mathrm{FVC}$ & $74.30 \pm 6.25$ & $82.75 \pm 1.67$ & $<0.001$ \\
$\mathrm{FEV}$ (\% pred) & $95.23 \pm 14.87$ & $117.30 \pm 13.04$ & $<0.001$ \\
$\mathrm{FVC}$ (\% pred) & $107.79 \pm 15,35$ & $118.47 \pm 14.75$ & 0.059 \\
$\mathrm{PEF}$ (\% pred) & $102.64 \pm 16.87$ & $117.85 \pm 16.41$ & 0.017 \\
$\mathrm{FEF} 75$ (\% pred) & $93.09 \pm 26.37$ & $123.97 \pm 18.13$ & 0.001 \\
$\mathrm{FEF}_{50}$ (\% pred) & $63.91 \pm 16.64$ & $110.08 \pm 17.23$ & $<0.001$ \\
$\mathrm{FEF}_{25}$ (\% pred) & $49.40 \pm 13.48$ & $84.44 \pm 14.83$ & $<0.001$ \\
$\mathrm{FEF}_{25-75}$ (\% pred) & $60.18 \pm 14.96$ & $102.56 \pm 15.86$ & $<0.001$
\end{tabular}

$\mathrm{FEV}_{1}$ forced expiratory volume in one second; FVC forced vital capacity; PEF peak expiratory flow; $\mathrm{FEF}_{75} / \mathrm{FEF}_{50} / \mathrm{FEF}_{25} / \mathrm{FEF}_{25-75}$ forced expiratory flow at $75 \% / 50 \% / 25 \%$ / $25-75 \%$ of FVC, respectively; \% pred \% predicted

In "At Risk" for COPD group, RA muscle activation intensity was significantly greater during breathing with expiratory load when compared to without respiratory load $(p=0.016)$ (Fig. 1$)$.

In Healthy group, the activation intensity of EO $(p=0.008)$ and $\operatorname{TrA} / \mathrm{IO}(p=0.039)$ muscles was significantly greater during breathing with expiratory load when compared to without respiratory load (Fig. 1).

\subsubsection{Expiration}

During expiration, no significant differences were found in the activation intensity of all abdominal muscles between "At Risk" for COPD and Healthy groups during all tasks (Fig. 2).

In "At Risk" for COPD group, the activation intensity of all abdominal muscles was significantly greater during breathing with expiratory load when compared to without respiratory load (RA: $p$ < 0.001; EO: $p=0.037$; TrA/IO: $p=0.014$ ). RA muscle activation intensity was significantly greater during breathing with inspiratory load when compared to without respiratory load $(p=0.009)$ (Fig. 2).

In Healthy group, the activation intensity of all abdominal muscles was significantly greater during breathing with expiratory load when compared to without respiratory load (RA: $p=0.008$; EO: $p=0.002$; $\operatorname{TrA} / \mathrm{IO}: p=0.007)$. TrA/IO muscle activation intensity was significantly greater during breathing with inspiratory load when compared to without respiratory load $(p=0.025)$. The activation intensity of all abdominal muscles was significantly greater during breathing with expiratory load when compared to inspiratory load (RA: $p=0.035$; EO: $p=0.038$; $\operatorname{TrA} / \mathrm{IO}: p=0.007$ ) (Fig. 2).

\subsubsection{Effect size}

Table 3 show the $\eta_{p}^{2}$, effect size and power test for the comparison of the percentage of activation intensity of RA, EO and $\operatorname{Tr} \mathrm{A} / \mathrm{IO}$ muscles between the different tasks, during inspiration and expiration, in "At Risk" for COPD and Healthy groups. During inspiration, in "At Risk" for COPD group, the comparison between the different tasks showed a large effect in RA muscle activation intensity $\left(\eta_{p}^{2}=0.37\right)$; in Healthy group, a large effect was observed in all abdominal muscles $\left(\eta_{p}^{2}>0.14\right)$ Still, during expiration, the comparison between the different tasks showed a large effect in the activation intensity of all abdominal muscles, in both groups $\left(\eta_{p}^{2}>0.14\right)$.

\section{Discussion}

Data of the present study suggested that subjects "at risk" for the development of COPD had a different recruitment pattern of 


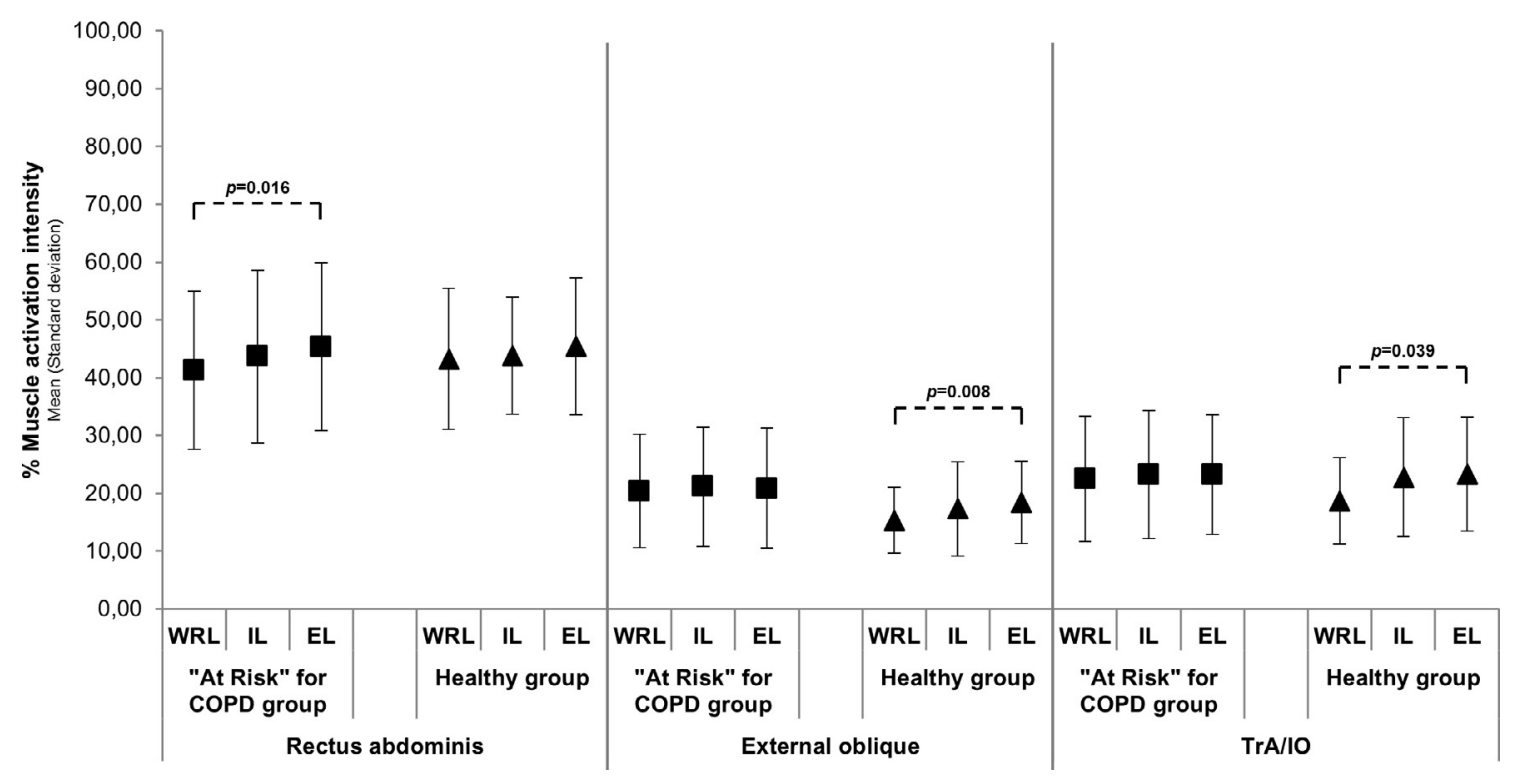

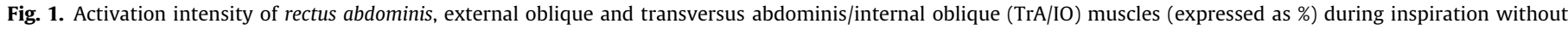

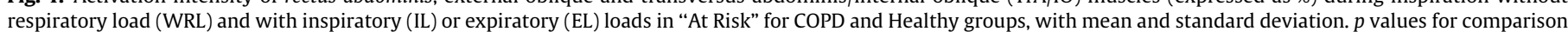
within subjects is also presented.

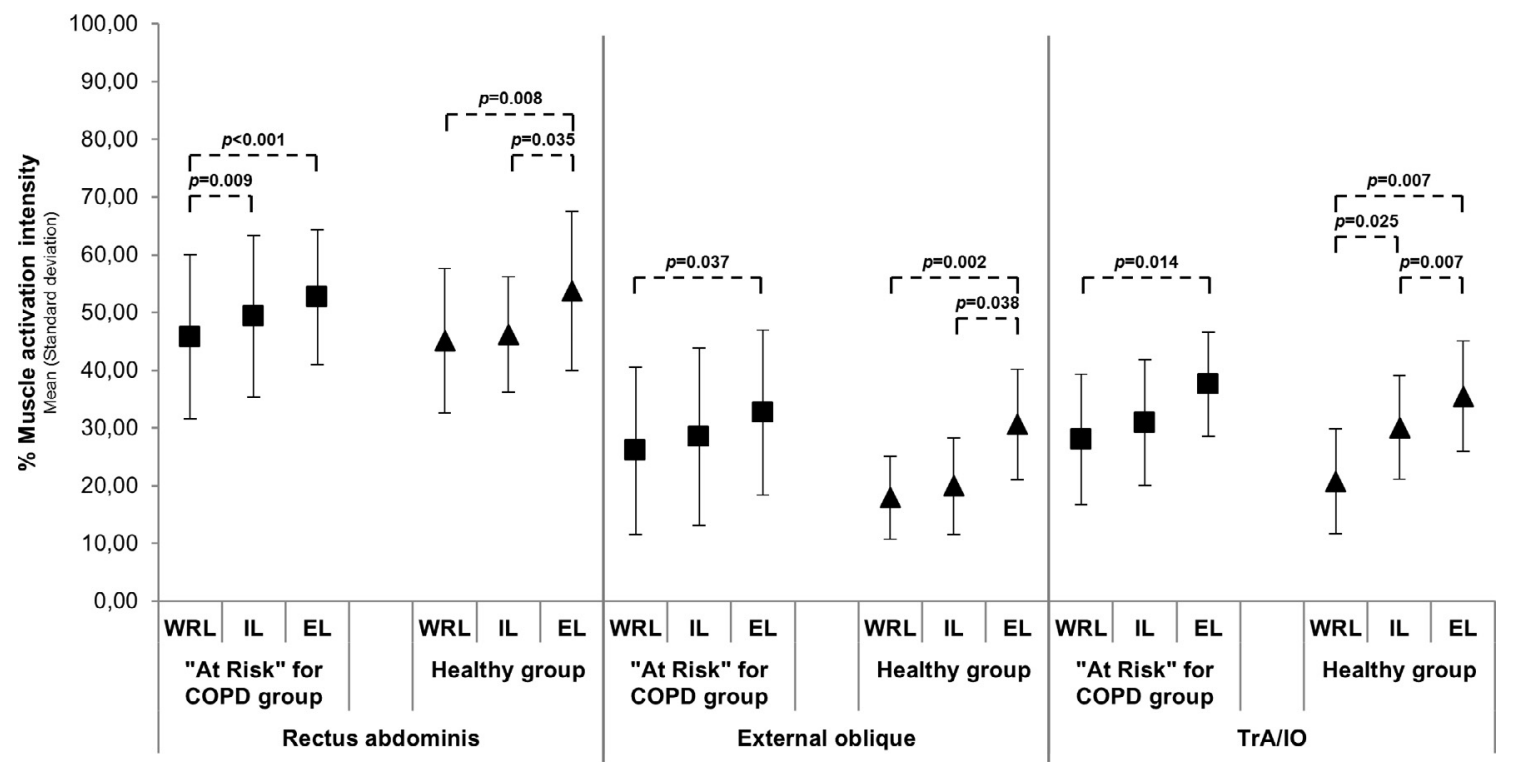

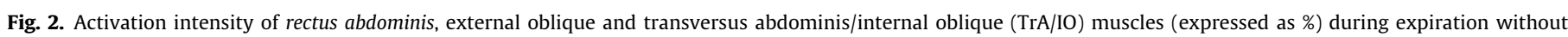

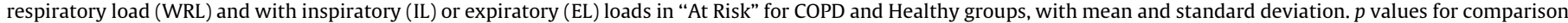
within subjects is also presented.

Table 3

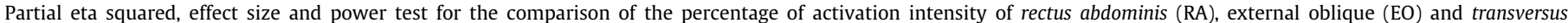

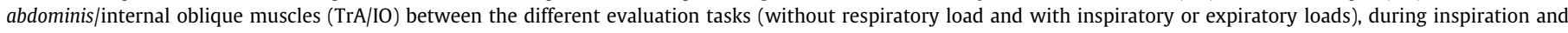
expiration, in "At Risk" for COPD and Healthy groups.

\begin{tabular}{|c|c|c|c|c|c|c|c|}
\hline \multirow[t]{2}{*}{ Breathing phase } & \multirow[t]{2}{*}{ Muscle } & \multicolumn{3}{|c|}{ “At Risk" for COPD group } & \multicolumn{3}{|l|}{ Healthy group } \\
\hline & & Partial eta squared & Effect size & Power test & Partial eta squared & Effect size & Power test \\
\hline \multirow[t]{3}{*}{ Inspiration } & $\mathrm{RA}$ & 0.376 & 0.776 & 1.000 & 0.169 & 0.451 & 0.946 \\
\hline & EO & 0.065 & 0.264 & 0.617 & 0.323 & 0.691 & 1.000 \\
\hline & TrA/IO & 0.034 & 0.188 & 0.347 & 0.376 & 0.776 & 1.000 \\
\hline \multirow[t]{3}{*}{ Expiration } & RA & 0.475 & 0.951 & 1.000 & 0.529 & 1.060 & 1.000 \\
\hline & EO & 0.285 & 0.631 & 1.000 & 0.563 & 1.135 & 1.000 \\
\hline & TrA/IO & 0.378 & 0.780 & 1.000 & 0.725 & 1.624 & 1.000 \\
\hline
\end{tabular}


abdominal muscles for the mechanics of breathing. This finding is important to furthering understand the natural history of COPD.

The quiet expiration is a passive process, involving the use of elastic components recoil of the lungs and chest wall; no phasic activity of abdominal muscles is observed (Kenyon et al., 1997). However, in this study, all participants breathed at a mandatory rhythm that increased the minute ventilation, implying a breathing below functional residual capacity and, consequently, a phasic respiratory activity of abdominal muscles. Moreover, the standardization of respiratory rhythm may have reduced the effect of different respiratory loads on minute ventilation, as well as breathing rate and pattern.

The findings of the present study indicated that there were no significant differences between "At Risk" for COPD and Healthy groups in activation intensity of all abdominal muscles during breathing without respiratory load. Evidence has been described that the abdominal muscle recruitment is frequent in COPD subjects (Ninane et al., 1992, 1993). Ninane et al. (1992) reported that, when breathing at rest, the phasic expiratory activation mainly concerns the $\operatorname{Tr} A$ muscle and its recruitment is related to the degree of airflow obstruction. Despite there was a decreased pulmonary function in "At Risk" for COPD group when compared to Healthy group, it seems that the differences do not reflect an obstructive ventilatory defect (Global Initiative for Chronic Obstructive Lung Disease, 2016; Rodriguez-Roisin et al., 2016). The variables associated with airflow obstruction (Tiffeneau index, $\mathrm{FEV}_{1}$ and $\mathrm{FEF}_{25-75 \%}$ ), in the study' sample, were above the cut-off points that define the limit of normality (Global Initiative for Chronic Obstructive Lung Disease, 2016; Rodriguez-Roisin et al., 2016). Thus, the breathing without respiratory load was not sufficiently challenging for the mechanics of breathing in these specific subjects. However, due to inspiratory and expiratory loads, the respiratory demand increases and requires voluntary control, promoting a different recruitment pattern of abdominal muscles within the groups.

\subsection{Inspiration}

In "At Risk" for COPD group, during breathing with expiratory load, the RA muscle activation intensity was greater when compared to without respiratory load, with a large effect; however, in Healthy group, the activation intensity of EO and TrA/IO muscles during breathing with expiratory load was greater than without respiratory load, during inspiration, with a large effect. Breathing is achieved by an alternate modulation of activity of the diaphragm and TrA muscles, resulting on cyclic changes in the shape of pressurized abdominal cavity, to support ribcage and abdominal movements (Hodges and Gandevia, 2000). The reduced abdominal wall compliance, via an increase in tonic contraction of abdominal muscles, would prevent further descent of the central tendon of diaphragm muscle and its effectively stabilization promotes lateral chest wall expansion (De Troyer and Estenne, 1988). The results of this study were consistent with earlier study of Gothe and Cherniack (1980). The altered recruitment pattern of abdominal muscles within the groups, there may have different consequences. The TrA/IO muscle activity, observed in Healthy group, may increase the transverse diameter of the lower rib cage (Key, 2013). Otherwise, in "At Risk" for COPD group, the RA muscle recruitment helps to anchor the thorax caudally and their excessive activity may constrict the inferior thorax, interfering with diaphragm descent (Key, 2013). The specific recruitment of abdominal muscles observed in each group may provide information relative to an impaired mechanics of breathing in subjects who have dyspnea, chronic cough or sputum production, and a history of exposure to risk factors for the chronic obstructive pulmonary disease (as tobacco smoke), but did not exhibited an obstructive ventilatory defect (Rodriguez-Roisin et al., 2016).

\subsection{Expiration}

In "At Risk" for COPD group, during with inspiratory load, the RA muscle activation intensity during breathing with inspiratory load was greater when compared to without respiratory load, during expiration, with a large effect; however, in healthy group, $\operatorname{Tr} \mathrm{A} /$ IO muscle activation intensity during breathing with inspiratory load was greater than without respiratory load, with a large effect. Due to loaded inspiration, it was expected, in both groups, a greater abdominal muscle activity during expiration. The respiratory system tends to limit the inspiratory muscle activity, transferring any additional load to the expiratory muscles, optimizing the length-tension relationship of diaphragm muscle fibres, to assist the subsequent inspiration (Aliverti et al., 1997). In Healthy group, the TrA/IO muscle expiratory contraction, due to its circumferential arrangement, should be more effective in increasing abdominal pressure and deflating the lung so as to take on portion the work of the inspiratory muscles (De Troyer et al., 1990). While TrA/IO muscle recruitment is beneficial in healthy subjects, the RA muscle expiratory contraction, observed in "At Risk" for COPD group, may be definitively harmful in an advanced pathological context. The inability of subjects with expiratory flow limitation to increase tidal expiratory flow rates and reduce end-expiratory lung volume below the resting value, or to induce the storage of elastic and gravitational energy in the diaphragm muscle and the abdomen (O'Donnell, 2001), by increasing expiratory activity of TrA/IO muscle, may place the diaphragm under a mechanical disadvantage. In both groups, all abdominal muscles showed a greater activation intensity with expiratory load when compared to without respiratory load, during expiration, with a large effect. Voluntary efforts, such as empty the lung or raise the intra-abdominal pressure, are achieved by the contraction in concert of the superficial (RA and EO muscles) and deep ( TrA/IO muscle) muscle layers of ventrolateral abdominal wall (De Troyer et al., 1990). These findings are corroborated by Mesquita Montes et al. (2016).

\subsection{Future investigation}

Further studies are needed to evaluate the impact of this altered recruitment pattern of abdominal muscles, observed in subjects "at risk" for the development of COPD, on the diaphragm function and its mechanics. The magnetic resonance imaging is a valid method to analyse the diaphragm motion and shape, during breathing. As the work of breathing may be increased in RA muscle' recruiters, an imbalance between energy supply and demand may result in competition between respiratory and other muscles for limited energy supplies (Aliverti and Macklem, 2008). Aliverti and Macklem (2008) reported that, as COPD progresses, subjects eventually learn to not recruit the abdominal muscles, minimizing the work and cost of breathing. Future studies are required to understand the contribution of specific recruitment of the superficial muscle layer of ventrolateral abdominal wall on the work and cost of breathing, in these subjects.

As another point of view, this recruitment pattern of superficial abdominal muscles during breathing with respiratory loads, observed in subjects "At Risk" for the development of COPD, may impair the postural control. The excessive trunk muscle activity, namely abdominal muscles, in response to increased respiratory demand, can compromise the trunk moments/movements (Smith et al., 2016). Therefore, the reduced contribution of trunk movement to postural control may be the source to balance deficits that were reported in COPD subjects (Janssens et al., 2013; Smith et al., 2010). Accordingly, further investigation is needed in order to understand the impact of the synergy of abdominal muscles and other trunk muscles for the postural control and mechanics of breathing, in subjects "at risk" for the development of COPD. 


\section{Conclusion}

The inspiratory and expiratory loads promoted a different impact on the abdominal muscle activity within each group. Subjects "at risk" for development of COPD seemed to have a specific recruitment pattern of the superficial muscle layer of ventrolateral abdominal wall (RA muscle) wherein the respiratory demand increases.

\section{References}

Aliverti, A., Cala, S.J., Duranti, R., Ferrigno, G., Kenyon, C.M., Pedotti, A., et al., 1997. Human respiratory muscle actions and control during exercise. J. Appl. Physiol. $83,1256-1269$.

Aliverti, A., Macklem, P.T., 2008. The major limitation to exercise performance in COPD is inadequate energy supply to the respiratory and locomotor muscles. J. Appl. Physiol. 105, 749-751 (discussion 55-7).

American Thoracic Society/European Respiratory, 2002. S. ATS/ERS statement on respiratory muscle testing. Am. J. Respir. Crit. Care Med. 166, 518-624.

Baraldo, S., Turato, G., Saetta, M., 2012. Pathophysiology of the small airways in chronic obstructive pulmonary disease. Respiration 84, 89-97.

Beith, I.D., Synnott, R.E., Newman, S.A., 2001. Abdominal muscle activity during the abdominal hollowing manoeuvre in the four point kneeling and prone positions. Man Ther. 6, 82-87.

Chanthapetch, P., Kanlayanaphotporn, R., Gaogasigam, C., Chiradejnant, A., 2009. Abdominal muscle activity during abdominal hollowing in four starting positions. Man Ther. 14, 642-646.

Cohen, J., 1988. Statistical Power Analysis for the Behavioral Sciences. L. Erlbaum Associates, Hillsdale, NJ.

Criswell, E., 2011. Cram's Introduction to Surface Electromyography. Jones \& Bartlett Publishers, Sudbury, MA.

de Marco, R., Accordini, S., Cerveri, I., Corsico, A., Anto, J.M., Kunzli, N., et al., 2007. Incidence of chronic obstructive pulmonary disease in a cohort of young adults according to the presence of chronic cough and phlegm. Am. J. Respir. Crit. Care Med. 175, 32-39.

de Oca, M.M., Halbert, R.J., Lopez, M.V., Perez-Padilla, R., Talamo, C., Moreno, D. et al., 2012. The chronic bronchitis phenotype in subjects with and without COPD: the PLATINO study. Eur. Respir. J. 40, 28-36.

De Troyer, A., Estenne, M., 1988. Functional anatomy of the respiratory muscles. Clin. Chest Med. 9, 175-193.

De Troyer, A., Estenne, M., Ninane, V., Van Gansbeke, D., Gorini, M., 1990. Transversus abdominis muscle function in humans. J. Appl. Physiol. 68, 10101016.

De Troyer, A., Leeper, J.B., McKenzie, D.K., Gandevia, S.C., 1997. Neural drive to the diaphragm in patients with severe COPD. Am. J. Respir. Crit. Care Med. 155, 1335-1340.

Drake, J.D., Callaghan, J.P., 2006. Elimination of electrocardiogram contamination from electromyogram signals: an evaluation of currently used removal techniques. J. Electromyogr. Kinesiol. 16, 175-187.

Gandevia, S.C., Leeper, J.B., McKenzie, D.K., De Troyer, A., 1996. Discharge frequencies of parasternal intercostal and scalene motor units during breathing in normal and COPD subjects. Am. J. Respir. Crit. Care Med. 153, 622-628.

Gea, J., Pascual, S., Casadevall, C., Orozco-Levi, M., Barreiro, E., 2015. Muscle dysfunction in chronic obstructive pulmonary disease: update on causes and biological findings. J. Thorac. Dis. 7, E418-E438.

Global Initiative for Chronic Obstructive Lung Disease. Global Strategy for Diagnosis, Management, and Prevention of Chronic Obstructive Pulmonary Disease - Update 2016. <http://goldcopd.org/global-strategy-diagnosismanagement-prevention-copd-2016/2016>.

Gorini, M., Spinelli, A., Ginanni, R., Duranti, R., Gigliotti, F., Scano, G., 1990. Neural respiratory drive and neuromuscular coupling in patients with chronic obstructive pulmonary disease (COPD). Chest 98, 1179-1186.

Gothe, B., Cherniack, N.S., 1980. Effects of expiratory loading on respiration in humans. J. Appl. Physiol. Respir. Environ. Exerc. Physiol. 49, 601-608.
Hermens, H.J., Freriks, B., Disselhorst-Klug, C., Rau, G., 2000. Development of recommendations for SEMG sensors and sensor placement procedures. J. Electromyogr. Kinesiol. 10, 361-374.

Hodges, P.W., Gandevia, S.C., 2000. Changes in intra-abdominal pressure during postural and respiratory activation of the human diaphragm. J. Appl. Physiol. 89, 967-976.

Janssens, L., Brumagne, S., McConnell, A.K., Claeys, K., Pijnenburg, M., Burtin, C. et al., 2013. Proprioceptive changes impair balance control in individuals with chronic obstructive pulmonary disease. PLoS One 8, e57949.

Kenyon, C.M., Cala, S.J., Yan, S., Aliverti, A., Scano, G., Duranti, R., et al., 1997. Rib cage mechanics during quiet breathing and exercise in humans. J. Appl. Physiol. 83 $1242-1255$.

Key, J., 2013. 'The core': understanding it, and retraining its dysfunction. J. Bodyw. Mov. Ther. 17, 541-559.

Laveneziana, P., Webb, K.A., Wadell, K., Neder, J.A., O’Donnell, D.E., 2014. Does expiratory muscle activity influence dynamic hyperinflation and exertional dyspnea in COPD? Respir. Physiol. Neurobiol. 199, 24-33.

Mannino, D.M., Doherty, D.E., Buist, A.S., 2006. Global Initiative on Obstructive Lung Disease (GOLD) classification of lung disease and mortality: findings from the Atherosclerosis Risk in Communities (ARIC) study. Respir. Med. 100, 115-122.

Marôco, J., 2014. Análise Estatística com o SPSS Statistics. sixth ed., Pero Pinheiro: ReportNumber, Lda

Marshall, P., Murphy, B., 2003. The validity and reliability of surface EMG to assess the neuromuscular response of the abdominal muscles to rapid limb movement. J. Electromyogr. Kinesiol. 13, 477-489.

Martinez, F.J., Couser, J.I., Celli, B.R., 1990. Factors influencing ventilatory muscle recruitment in patients with chronic airflow obstruction. Am. Rev. Respir. Dis. $142,276-282$.

Mesquita Montes, A., Baptista, J., Crasto, C., de Melo, C.A., Santos, R., Vilas-Boas, J.P. 2016. Abdominal muscle activity during breathing with and without inspiratory and expiratory loads in healthy subjects. J. Electromyogr. Kinesiol. 30, 143-150.

Mew, R., 2009. Comparison of changes in abdominal muscle thickness between standing and crook lying during active abdominal hollowing using ultrasound imaging. Man Ther. 14, 690-695.

Miller, M.R., Hankinson, J., Brusasco, V., Burgos, F., Casaburi, R., Coates, A., et al. 2005. Standardisation of spirometry. Eur. Respir. J. 26, 319-338.

Ninane, V., Rypens, F., Yernault, J.C., De Troyer, A., 1992. Abdominal muscle use during breathing in patients with chronic airflow obstruction. Am. Rev. Respir. Dis. $146,16-21$.

Ninane, V., Yernault, J.C., de Troyer, A., 1993. Intrinsic PEEP in patients with chronic obstructive pulmonary disease. Role of expiratory muscles. Am. Rev. Respir. Dis. 148, 1037-1042.

O'Donnell, D.E., 2001. Ventilatory limitations in chronic obstructive pulmonary disease. Med. Sci. Sports Exerc. 33, S647-S655.

Reeve, A., Dilley, A., 2009. Effects of posture on the thickness of transversus abdominis in pain-free subjects. Man Ther. 14, 679-684.

Rodriguez-Roisin, R., Han, M.K., Vestbo, J., Wedzicha, J.A., Woodruff, P.G., Martinez, F.J., 2016. Chronic respiratory symptoms with normal spirometry: a reliable clinical entity? Am. J. Respir. Crit. Care Med.

Smith, M.D., Chang, A.T., Hodges, P.W., 2016. Balance recovery is compromised and trunk muscle activity is increased in chronic obstructive pulmonary disease. Gait Posture. 43, 101-107.

Smith, M.D., Chang, A.T., Seale, H.E., Walsh, J.R., Hodges, P.W., 2010. Balance is impaired in people with chronic obstructive pulmonary disease. Gait Posture. $31,456-460$.

Stavem, K., Sandvik, L., Erikssen, J., 2006. Can global initiative for Chronic Obstructive Lung Disease stage 0 provide prognostic information on longterm mortality in men? Chest 130, 318-325.

Thompson, P.D., 2014. Health appraisal and risk assessment. In: Pescatello, L.S. Arena, R., Riebe, D., Thompson, P.D. (Eds.), ACSM's Guidelines for Exercise Testing and Prescription. sixth ed. Wolters Kluwer/Lippincott Williams \& Wilkins Health, Philadelphia, pp. 1-18.

Vestbo, J., Hurd, S.S., Agusti, A.G., Jones, P.W., Vogelmeier, C., Anzueto, A., et al., 2013. Global strategy for the diagnosis, management, and prevention of chronic obstructive pulmonary disease: GOLD executive summary. Am. J. Respir. Crit. Care Med. 187, 347-365. 